\title{
Flora das cangas da Serra dos Carajás, Pará, Brasil: Phyllanthaceae
}

\author{
Flora of the cangas of the Serra dos Carajás, Pará, Brasil: Phyllanthaceae
}

Ricardo de S. Secco ${ }^{1,3}$ \& João Barros da Silveira ${ }^{1}$

\begin{abstract}
Resumo
Um tratamento taxonômico para as espécies de Phyllanthaceae das cangas da Serra dos Carajás, no estado do Pará, é apresentado. O estudo foi baseado em coleções depositadas nos Herbários BHCB, IAN, INPA, MG e NY, além de amostras observadas no campo. Foram registradas cinco espécies de Phyllanthus, como seguem: Phyllanthus carolinensis, P. hyssopifoliodes, P. minutulus, P. orbiculatus e Phyllanthus stipulatus. São apresentadas descrições, chave taxonômica, ilustrações e comentários para as espécies.

Palavras-chave: Amazônia, Euphorbiaceae, Phyllanthus, taxonomia.
\end{abstract}

\begin{abstract}
A taxonomic treatment of the species of Phyllanthaceae from the cangas (areas of iron-rich soils) of Serra dos Carajás, in Pará state, is presented. The study was based on collections deposited in the herbaria BHCB, IAN, INPA, MG and NY, as well as samples observed in the field. Five species of Phyllanthus were recorded (Phyllanthus carolinensis, P. hyssopifoliodes, P. minutulus, P. orbiculatus and Phyllanthus stipulates). Descriptions, a taxonomic key, illustrations and comments about the species are provided.
\end{abstract}

Key words: Amazon, Euphorbiaceae, Phyllanthus, taxonomy.

\section{Phyllanthaceae}

Phyllanthaceae Martinov abrange cerca de 59 gêneros e 2.000 espécies, com distribuição pantropical (Hoffmann et al. 2006)). De acordo com BFG (2015), no Brasil ocorrem 14 gêneros e cerca de 118 espécies, a maioria pertencente a Phyllanthus, gênero com um total aproximado de 88 espécies, encontradas em todas as regiões do país. As Phyllanthaceae são ervas, arbustos ou árvores, com folhas alternas, inflorescências cimosas, axilares; flores unissexuadas em plantas monoicas ou dioicas, sépalas livres ou conatas, pétalas ausentes ou presentes (reduzidas), livres ou conatas, ovário súpero e fruto esquizocarpo (Judd et al. 2008). Entre as características principais que a diferenciam das Euphorbiaceae estão a ausência de látex, dois óvulos em cada lóculo do ovário e sementes desprovidas de arilo (Judd et al. 2008; Secco \& Rosário 2015). Na Serra dos Carajás foram registradas cinco espécies de Phyllanthus. Do ponto de vista taxonômico, as Phyllanthaceae ainda são pouco estudadas na Amazônia brasileira, especialmente as espécies de Phyllanthus ocorrentes em áreas não florestais.

\section{Phyllanthus L., Sp. Pl. 981. 1753.}

Phyllanthus é representado especialmente por ervas, também arbustos, menos frequente árvores, monoicos, raramente dioicos, com ramificação filantoide ou não filantoide; folhas alternas, inteiras, inflorescências cimosas, axilares, às vezes representadas por uma única flor, flores monoclamídeas, unissexuadas, gamossépalas, lobos discretamente unidos na base, geralmente com disco inteiro ou segmentado; flores estaminadas com cálice 5-6 lobos, estames 2-6, livres ou unidos, anteras com deiscência vertical, oblíqua ou horizontal; flores pistiladas com cálice 4-6 lobos, ovário 3-locular; fruto cápsula, sementes trígonas, sem carúncula. Gênero ainda mal interpretado na Amazônia, especialmente as formas herbáceas, cujas flores estaminadas são reduzidas, às vezes difíceis de analisar em material herborizado. A forma das folhas também varia em muitas dessas espécies, algumas parecendo representar "complexo de espécies", ou então complexo de híbridos (Webster 2002), levando a equívocos que as identificam sempre como $P$. niruri L.

\footnotetext{
Museu Paraense Emilio Goeldi, Av. Magalhães Barata 376, São Braz, 66040-170, Belém, PA, Brasil

${ }^{3}$ Autor para correspondência: rsecco@museu-goeldi.br
} 


\section{Chave de identificação das espécies de Phyllanthus das cangas da Serra dos Carajás}

1. Ramos filantoides

2. Folhas obovais, ovais, elíptico-lanceoladas a elíptico-oblongas; cálice 5-lobado; estames 2-3 unidos em coluna.

3. Folhas obovais a ovais; estames 2 .

1.3. Phyllanthus minutulus

3'. Folhas elíptico-lanceoladas a elíptico-oblongas; estames 3.

1.5. Phyllanthus stipulatus

2'. Folhas orbiculares; cálice 6-lobado; estames 3, unidos apenas na base

1.4. Phylanthus orbiculatus

1'. Ramos não filantoides

4. Folhas elíptico-lanceoladas, adpressas nos ramos, dispostas como "escamas"; estiletes patentes, disco da flor pistilada segmentado .....

1.2. Phyllanthus hyssopifolioides

4'. Folhas elípticas a elíptico-ovais, sem essa característica; estiletes eretos, disco da flor pistilada inteiro 1.1. Phyllanthus carolinensis

1.1. Phyllanthus carolinensis Walter, Fl. Carol. 228. 1788 .

Fig. 1a-c

Erva a subarbusto $5-50 \mathrm{~cm}$ alt. Ramos não filantoides, glabros. Folhas alternas, elípticas a elíptico-ovais, $0,8-1,5 \times 0,4-0,7 \mathrm{~cm}$, membranáceas, glabras ou ocasionalmente com tricomas simples esparsos na face adaxial, base cuneada, ápice arredondado, agudo a obtuso, às vezes discretamente acuminado, margem inteira, nervuras bem evidentes; pecíolo $1-1,5 \mathrm{~mm}$ compr., glabro. Inflorescência com flores aos pares, estaminada 1, acompanhada de uma pistilada, axilares. Flores estaminadas pedicelo 1,2-1,5 mm compr., glabro; cálice 6-lobado, lobos ovais, 1-1,5 mm compr., glabros; estames 3 , livres, anteras com deiscência horizontal, disco segmentado. Flores pistiladas pedicelo $0,8-1 \mathrm{~mm}$ compr., glabro; cálice 6-lobado, lobos elíptico-lanceolados, 0,9-1 mm compr.; ovário globoso, $0,3-0,5$ mm diâm., discretamente muricado, estiletes 3, eretos, ramos bífidos até a metade, disco inteiro, cupuliforme. Cápsula, 1,5-2 mm diâm., subglobosa a depresso-ovalada, glabra. Sementes $0,8-1 \mathrm{~mm}$ compr.,trígonas, discretamente verruculosas.

Material selecionado: Marabá, Serra dos Carajás, $5^{\circ} 53^{\prime} \mathrm{S}$, 50³0'W', 16.VII.1984, N.A. Rosa et al 4618 (MG).

Material adicional: BRASIL. PARÁ: Oriximiná, 11.VI.1957, W.A. Egler 551 (MG); Belém, Jardim do Colégio Gentil Bittencourt, 30.IV.1970, P. Cavalcante 2702 (MG); Itaituba, Parque Nacional do Tapajós, Km 60 Estrada Itaituba-Jacareacanga, 22.XI.1978, M.G.Silva \& C. Rosario 3895 (INPA, MG).

Phyllanthus carolinensis pode ser confundida com P. tenellus Roxb., porém esta possui ramos filantoides (vs. ramos não filantoides), flor pistilada com pedicelo 2-7 $\mathrm{mm}$ compr. (vs. flor pistilada com pedicelo $0,8-1 \mathrm{~mm}$ ), cálice 5-lobado (vs. cálice 6-lobado) e estames 5 (vs. estames 3).
Espécie de ampla distribuição na região neotropical, desde os Estados Unidos, Antilhas até a Argentina (Silva \& Sales 2007). Segundo BFG (2015), no Brasil ocorre no Norte (Amapá, Pará, Amazonas, Acre e Rondônia), Nordeste (Pernambuco e Bahia), Centro-Oeste (Goiás), Sudeste (Espirito Santo, Minas Gerais, Rio de Janeiro e São Paulo) e Sul (Paraná e Santa Catarina). Na Serra dos Carajás, até o momento foi registrada apenas na vegetação ribeirinha, próxima ao Rio Itacaiúnas, a 45 km de N1 (entre N1 e mina de cobre do Salobo).

\subsection{Phyllanthus hyssopifolioides Kunth, Nov.} Gen. Sp. Pl. 2: 108.1817. Fig. 1d-g

Erva ou subarbusto 5-40 cm alt., glabros. Ramos não filantoides, glabros. Folhas alternas, subsésseis, adpressas nos ramos (dispostas como "escamas"), elíptico-lanceoladas, $0,5-1 \times 0,15-0,3$ $\mathrm{cm}$, cartáceas, base cuneada a atenuada, ápice agudo a discretamente acuminado, margem inteira, glabras em ambas as faces; pecíolo $1 \mathrm{~mm}$ compr., glabro. Inflorescência com flores dispostas nas axilas das folhas, as estaminadas duas a três, as pistiladas isoladas ou aos pares. Flores estaminadas pedicelo $0,8-1 \mathrm{~mm}$ compr., filiforme, glabro; cálice 6-lobado, lobos orbiculares, 0,5-07 mm compr., glabros; estames 3 , livres, anteras com deiscência lateral, disco segmentado, segmentos livres. Flores pistiladas pedicelo $0,5-0,7 \mathrm{~mm}$ compr., arredondado, glabro; cálice 6-lobado, lobos elíptico-ovais, 1-1,5 mm compr., 1,5 mm larg., glabros; ovário 1-1,5 mm diâm., subgloboso, glabro, estiletes 3, patentes, disco segmentado. Cápsula 1,8-2 mm diâm., glabra. Sementes 0,8-1,5 $\mathrm{mm}$ compr., trígonas, verruculosas.

Material selecionado: Parauapebas, Serra Norte, N4, 700-750 m, 15.III.1984, A.S.L. da Silva et al. 1805 

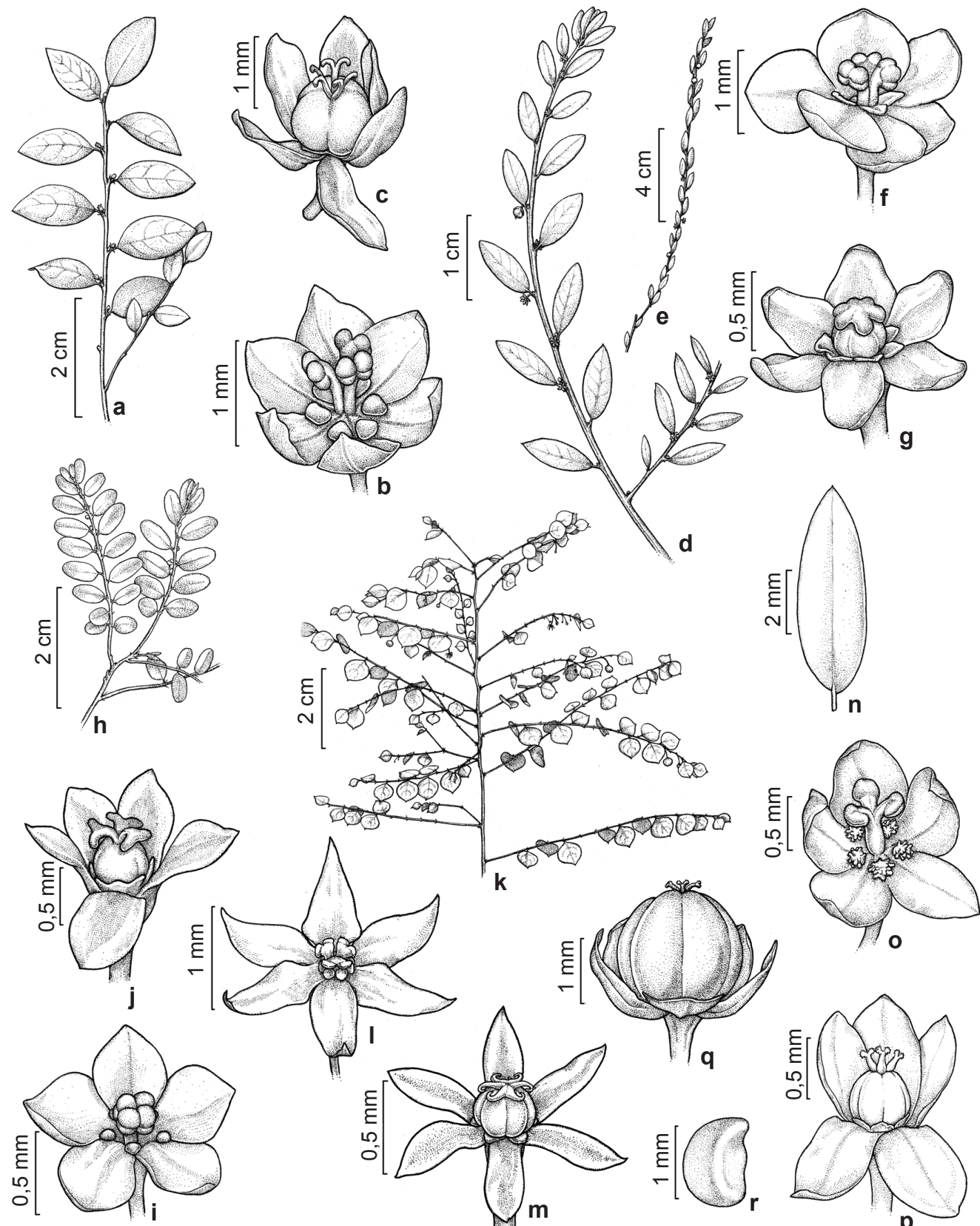

Figura 1 - a-c. Phyllanthus carolinensis - a. ramo, b. flor estaminada, c. flor pistilada; d-g. Phyllanthus hyssopifolioides. -d. ramo, e. ramo, folhas menores, f. flor estaminada, g. flor pistilada; $\mathrm{h}-\mathrm{j}$. Phyllanthus minutulus - h. ramo, i. flor estaminada, j. flor pistilada; k-m. Phyllanthus orbiculatus - k. Ramo, 1. flor estaminada, m. flor pistilada; n-r. Phyllanthus stipulatus - n. folha, o. flor estaminada, p. flor pistilada, q. fruto, r. semente. (a-c: N.A. Rosa et al. 4618; d-g: O.C. Nascimento \& R.P. Bahia 1063; h-j: P. Cavalcante 2625; k-m: D.C. Daly et al. 1791; n-r: A.S.L. Silva 1805).

Figure 1 - a-c. Phyllanthus carolinensis - a. branch, b. staminate flower, c. pistillate flower; d-g. Phyllanthus hyssopifolioides - d. branch, e. branch with small leaves, f. staminate flower, g. pistillate flower; $\mathrm{h}-\mathrm{j}$. Phyllanthus minutulus $-\mathrm{h}$. branch, i. staminate flower, j. pistillate flower; $\mathrm{k}-\mathrm{m}$. Phyllanthus orbiculatus - k. branch, l. staminate flower, m. pistillate flower. n-r. Phyllanthus stipulatus - n. leaf, o. staminate flower, p. pistillate flower, q. fruit, r. seed (a-c: N.A. Rosa et al. 4618; d-g: O.C. Nascimento \& R.P. Bahia 1063; h-j: P. Cavalcante 2625; k-m: D.C. Daly et al. 1791; n-r:A.S.L. Silva 1805). 
(MG); Serra Norte, N3, Lagoa, 702 m, 602'30"S, $50^{\circ} 12$ '26"W, 29.III.2015, R.M. Harley et al. 57148 (MG); próximo de N5, 6 02 ' $25^{\prime}$ 'S , 5005'17' $\mathrm{W}$, 30.IV.2015, A. Gil 495 (MG).

Espécie muito típica pelas folhas subsésseis, adpressas nos ramos, dispostas como "escamas", característica especialmente observada no material herborizado. Algumas vezes confundida $\operatorname{com} P$. carolinensis em identificações encontradas nos herbários, porém $P$. hyssopifolioides se destaca pela disposição das folhas ao longo dos ramos, lobos do cálice da flor estaminada orbiculares (vs. lobos do cálice da flor estaminada ovais), estiletes patentes (vs. estiletes eretos) e disco da flor pistilada segmentado (vs. disco inteiro). Não havia sido registrada em nenhum tratamento taxonômico de Phyllanthus já publicado no Brasil.

No Brasil ocorre em Roraima, no Amapá (novo registro), Pará, Amazonas, Goiás e Paraná, nas matas, campos e cerrados. Na Serra dos Carajás foi coletada na Serra Norte: N3, N4, N4, Serra da Bocaina (Serra do Rabo) e Serra do Tarzan, geralmente encontrada em áreas alagadas, beira de lago e brejos sobre canga.

\subsection{Phyllanthus minutulus Müll. Arg., Fl. Bras.} 11(2): 54. 1873.

Fig. $1 \mathrm{~h}-\mathrm{j}$

Erva a subarbusto $20-70 \mathrm{~cm}$ alt. Ramos filantóides. Folhas obovais a ovais, 0,25-1,2 $\times 0,15-0,3 \mathrm{~cm}$, base cuneada, ápice agudo, margem inteira, glabras; pecíolo 0,5-1 mm compr., glabro. Inflorescência com flores estaminadas 3, pistiladas isoladas. Flores estaminadas pedicelo 1-1,2 mm compr, glabro; cálice 5-lobado, lobos elíptico-ovais, 0,5-1 $\mathrm{mm}$ compr, 0,5-0,7 mm larg., glabros; estames 2, filetes concrescidos, formando coluna, anteras com abertura lateral, disco segmentado. Flores pistiladas pedicelo 0,5-1 mm compr., glabro, receptáculo cônico conspícuo; cálice 5-lobado, lobos elípticos, 0,5-1 mm, 0,5-1,2 mm larg., glabros; ovário 0,5-0,7 mm diâm., subgloboso, glabro, estiletes 3, bífidos, eretos, disco segmentado. Cápsula 1,5-2,5 mm diâm., glabra. Sementes 1-1,7 mm compr., trígonas, estriadas.

Material selecionado: Canãa dos Carajás, Serra Sul, Corpo D, 6024'13"S, 50¹8'17' W, 683 m, 18.II.2010, F.D. Gontijo 115 (BHCB); Serra Sul, 750 m, 08.XII.2007, P.L. Viana et al. 3422 (BHCB); Serra Sul, 6²1'6"S, $50^{\circ} 23$ '44"'W , 820 m, 14.II.2010, M.O. Pivari et al. 1472 (BHCB); Serra Sul, S11B, 738 m, 02.XII.2015, J.L.C. Costa 29 (MG); Serra da Bocaina, 6 17 '46", S, 4954'34'W, 08.III.2012, L.C.V. Silva et al. 1225 (BHCB); Serra do Rabo-Norte, 6 ${ }^{\circ} 18$ '14'S, 4953'57' W,
716 m, 15.XII.2010, N.F.O. Mota et al. 1889 (BHCB); Serra do Tarzan, 6²0'10" S, 5009'48' W, 700 m, 13.III.2009, P.L. Viana et al. 4039 (BHCB). Parauapebas [Marabá]: Serra dos Carajás, N4, 700-750 m, 15.III.1984, A.S.L. da Silva 1807 (MG); Serra Norte, clareira N1, 18.IV.1970, P. Cavalcante 2625 et M. Silva (MG); N3, 27.04.2015, N.F.O. Mota et al. 2945 (MG); Serra Norte, prox. N5, 602'25''S, 5005'17'”W, 30.IV.2015, A. Gil 496 (MG); Platô N5, 6 05'23”S, 50¹1'33”W, 13.III.2015, L.C. Lobato 4317 (MG).

Phyllanthus minutulus compartilha características morfológicas com $P$. stipulatus (Raf.) G.L. Webster, tais como folhas reduzidas e cinco sépalas, porém destaca-se pela presença de dois estames (vs. três estames), flores pistiladas com pedicelo 0,5-1 mm compr. (vs.flores pistiladas com pedicelo 2,5-3 mm compr.), e folhas obovais a ovais (vs. folhas elíptico-lanceoladas a elípticooblongas). Mas de acordo com Webster (2002), $P$. minutulus possui dois estames, raramente três. E tal variação no número de estames parece requerer um estudo mais detalhado em relação a $P$. stipulatus, da qual provavelmente $P$. minutulus represente apenas uma variação (?) com dois estames.

Espécie amplamente distribuída na Colômbia, Venezuela, Guiana e Brasil, aqui ocorrendo de Norte ao Sul (BFG 2015). Na Serra dos Carajás foi coletada na Serra Norte: N1, N3, N4 e N5, Serra Sul: S11B, S11D, Serra da Bocaina (Serra do Rabo) e Serra do Tarzan. Ocorre em áreas úmidas e alagadas, brejos sobre canga e transição mata/ solo rupestre.

1.4. Phyllanthus orbiculatus Rich., Actes Soc. Hist. Nat. Paris 1: 113. $1792 . \quad$ Fig. 1k-m

Erva a subarbusto $5-80 \mathrm{~cm}$ alt. Ramos filantoides, glabros. Folhas alternas, 0,5-1 $\times$ 0,4-1,2 cm, orbiculares, muito delicadas, membranáceas, base arredondada, ápice agudo, margem inteira, glabras em ambas as faces; pecíolo muito reduzido, 0,5-0,7 $\mathrm{mm}$ compr., filiforme, glabro. Inflorescência com flores isoladas ou aos pares, dispostas ao longo dos ramos, às vezes nas axilas das folhas. Flores estaminadas pedicelo 2,5$3 \mathrm{~mm}$ compr., filiforme, glabro; cálice 6-lobado, lobos elíptico-lanceolados, transparentes, 1-1,2 mm compr., 0,5-0,7 mm larg., glabros, ápice discretamente acuminado; estames 3 , concrescidos na base; flores pistiladas pedicelo 1,5-2,5 mm compr., receptáculo cônico, bem evidente; cálice 6-lobado, lobos lanceolados, 0,5-0,6 mm compr., 0,1-0,2 mm larg., glabros; ovário 0,5-1 mm diâm., subgloboso, trilocular, glabro, estiletes 3 , bífidos, sésseis. Cápsula 1,2-1,8 mm diâm., 
mericarpos 6, glabros. Sementes 1,0-1,5 mm compr., verruculosas.

Material selecionado: Serra dos Carajás, $25 \mathrm{~km}$ of Serra Norte mining camp, aprox. 555'S, 50²6'W, 6.XII.1981, fl., D.Daly et al. 1791 (MG, NY); Canãa dos Carajás, Serra do Rabo, 6¹8'36'S, 4953'22'W, 692 m, 14.XII.2007, N.F.O. Mota et al. 1192 (BHCB); Parauapebas, Serra do Rabo-Norte, 6¹8'51'S, 495 54'16'W, 717 m, 19.XII.2010, N.F.O. Mota et al.1997 (BHCB).

Phyllanthus orbiculatus é inconfundível pelas folhas orbiculares, extremamente delicadas, membranáceas, que a diferenciam prontamente das demais espécies de Phyllanthus encontradas na Serra dos Carajás. Compartilha com P. minutulus o tipo de ramificação filantoide, porém, logo se destaca pelas flores com cálice 6-lobado (vs. flores com cálice 5-lobado) e 3 estames (vs. 2 estames).

Ocorre amplamente na América do Sul, abrangendo Colômbia, Venezuela, Trinidad, Guianas, Peru e Brasil (Webster et al.1999). Segundo BFG (2015), ocorre nas regiões de Norte a Sul do Brasil. Silva \& Sales (2007) a registram em Pernambuco. Habita em matas úmidas, savanas, áreas antropizadas e como ruderal. Cordeiro (1992) a registrou nos campos rupestres da Serra do Cipó, Minas Gerais. $\mathrm{Na}$ Serra dos Carajás, foi coletada na Serra Norte e Serra da Bocaina (Serra do Rabo), onde é encontrada geralmente em beira de mata e solo de canga.

1.5. Phyllanthus stipulatus (Raf.) G.L. Webster, Contr. Gray Herb. 176: 53. 1955.

Moeroris stipulata Raf., Sylva Tellur. 91. 1838.

Fig. 1n-r

Erva 15-50 cm alt. Ramos filantoides. Folhas 0,55-0,8 ×0,25-0,45 cm, elíptico-lanceoladas a elíptico-oblongas, ápice agudo, base arredondadas a discretamente cuneada, margem inteira; pecíolo 0,6-1 mm compr., glabro. Inflorescência com flores estaminadas 3, pistiladas isoladas na porção terminal dos ramos. Flores estaminadas pedicelo 1-1,5 mm compr., delicado, glabro; cálice 5-lobado, lobos ovais, glabros; estames 3 , unidos em coluna, anteras com deiscência horizontal, disco segmentado, verruculoso. Flores pistiladas pedicelo 2,5-3 mm compr.; cálice 5-lobado, lobos ovais, 0,6-1 mm compr., 0,7-0,8 mm larg., glabros; ovário subgloboso, 0,8-0,9 mm diâm., glabro, disco lobado.Cápsula 1,8-2,5 mm diâm., glabra. Sementes 1,0-1,6 mm compr., discretamente estriadas. Material selecionado: Marabá, Serra dos Carajás, N-4, 700-750 m, 15.III.1984, A.S.L. da Silva et al. 1805 (MG). Material adicional: BRASIL. PARÁ: Oriximiná, 02.VII.1980, C. Davidson 10693 (INPA, MG); Marituba, 27.X.1977, E. Oliveira 6707 (MG).
Phyllanthus stipulatus mantém afinidade com P. minutulus (ver comentários daquela espécie). No presente tratamento foi seguido o conceito que envolve número de estames, forma de folhas e variação do pedicelo para diferenciar as duas espécies. Porém, ambas fazem parte de um grupo de espécies muito próximas de Phyllanthus sect. Phyllanthus subsect. Swartziani (Webster 2002), que inclui também $P$. caribaeus Urb., P. lindbergii e P. microphyllus, requerendo uma revisão mais acurada sobre suas afinidades.

De acordo com BFG (2015) ocorre em todas as regiões brasileiras, abrangendo Roraima, Amazonas, Acre, Pará, Rondônia, Maranhão, Bahia, DF, Goiás, Mato Grosso do Sul, Mato Grosso, Espírito Santo, Minas Gerais, São Paulo, Paraná e Santa Catarina, em cerrado, campo rupestre, mata atlântica, mata de terra firme, igapó e várzea. Na Serra dos Carajás foi coletada na Serra Norte: N4, em campo rupestre (solo de canga).

\section{Agradecimentos}

Ao CNPq, a concessão de bolsa Pq (processo n. 305041/2013-4) ao primeiro autor para estudar as Phyllanthaceae da Amazônia, e de bolsa DTI ao co-autor. Aos coordenadores da Flora das cangas de Carajás, Dr. Pedro Viana e Dra. Ana Maria GiuliettiHarley, o convite para participar do projeto. Ao ITV-Instituto Tecnológico Vale (01205.000250/201410) e ao CNPq (processo n. 4555005/2014-4), o financiamento. Ao Dr. Nathan Smith, a elaboração do abstract.

\section{Referências}

BFG. 2015. Growing knowledge: an overview of seed plant diversity in Brazil. Rodriguésia 66: 1085-1113.

Cordeiro, I. 1992. Flora da Serra do Cipó, Minas Gerais: Euphorbiaceae. Boletim de Botânica, Universidade de São Paulo 13: 169-217.

Hoffmann, P.; Kathriarachchi, H. \& Wurdack, K.J. 2006. A phylogenetic classification of Phyllanthaceae (Malpighiales. Euphorbiaceae sensu lato). Kew Bulletin 61: 37-53.

Judd, W.S.; Campbell, C.S.; Kellog, E.A.; Stevens, P.F. \& Donoghue. 2008. Plant Systematics. $3^{\text {a }}$ ed. Sinauer Associates publishers, Sunderland. 464p.

Secco, R.S. \& Rosário, A.S. 2015. A new species of Phyllanthus (Phyllanthaceae) endemic to Amazonas state, Brazil. Novon 24: 209-211.

Silva, M.J. \& Sales, M.F. 2007. Phyllanthus L. (Phyllanthaceae) em Pernambuco, Brasil. Acta Botanica Brasilica 21: 79-98.

Webster, G.L.; Berry, P.E.; Armbruster, W.S.; Esser. H.-J.; Gillespie, L.J.; Hayden, W.J.; Levin, G.A.; Secco, R.S. \& Heald, S.V. 1999. Euphorbiaceae. In: Berry, P.E.; 
Yatskievych, K. \& Holts, B.K. (eds.). Flora of the Venezuelan Guayana. Vol 5. Missouri Botanical Garden Press, St. Louis. Pp 191-205.
Webster, G.L. 2002. A synopsis of the Brazilian taxa of Phyllanthus section Phyllanthus (Euphorbiaceae). Lundellia 5: 1-26.

\section{Lista de exsicatas}

Cavalcante, P. 2702 (1.1), 2625 (1.3); Costa, J.L.C. 29 (1.3); Costa, L.V. 1055 (1.2); Daly, D. 1698 (1.2), 1791 (1.4); Davidson, C. 10693 (1.5); Egler, W.A. 551 (1.1); Gil, A. 495 (1.2), 496 (1.3); Giorni, V.T. 141 (1.2), 222 (1.3); Gontijo, F.D. 115 (1.3); Harley, R.M. 57148 (1.2); Lobato, L.C. 4317 (1.3); Mota, N.F.O. 1889 (1.3), 2945 (1.3), 1192 (1.4), 1929 (1.2); 1997 (1.4); Nascimento, O.C. 1063 (1.2); Oliveira, E. 6707 (1.5); Pivari, M.O. 1472 (1.3); Rosa, N.A. 4618 (1.1); da Silva A.S.L. 1807 (1.3), 1805 (1.5); Silva L.C.V. 1225 (1.3); Silva, M.G. 3895 (1.1); Sperling, C.R. 6262 (1.2), 5701 (1.3); Viana, P.L. 3422 (1.3), 4039 (1.3), 3467 (1.2). 